\title{
Nouveau jalon pour un partenariat historique \\ Le Consortium régional de recherche en éducation du Saguenay-Lac-St-Jean : un nouveau jalon pour un partenariat historique
}

Auteur(s)

Stéphane Allaire, UQAC, Canada, stephane allaire@uqac.ca

Marie-Pierre Baron, UQAC, Canada, marie-pierre baron@uqac.ca

Souleymane Barry, UQAC, Canada, souleymane barry@uqac.ca

Nadia Cody, UQAC, Canada, nadia cody@uqac.ca

Carole Côté, UQAC, Canada, carole cote@uqac.ca

Sandra Coulombe, UQAC, Canada, sandra coulombe@uqac.ca

Christine Couture, UQAC, Canada, christine couture@uqac.ca

Catherine Dumoulin, UQAC, Canada, catherine dumoulin@uqac.ca

Diane Gauthier, UQAC, Canada, diane gauthier@uqac.ca

Patrick Giroux, UQAC, Canada, patrick giroux@uqac.ca

Raoul Kamga Kouamkam, UQAC, Canada, raoul.kamga-kouamkam.1@ulaval.ca

Catherine Larouche, UQAC, Canda, catherine larouche@uqac.ca

Nicole Monney, UQAC,Canada, nicole monney@uqac.ca

Sylvie Morais, UQAC, Canada, sylvie morais@uqac.ca

Loïc Pulido, UQAC, Canada, loic pulido@uqac.ca

Pascale Thériault, UQAC, Cadana, pascale theriault@uqac.ca 


\section{REVUE HYBRIDE DE L'ÉDUCATION}

\section{Résumé}

Ce texte rend compte, en premier lieu, de l'organisation d'un partenariat unique en éducation, le Consortium régional de recherche en éducation. Ensuite, nous expliquons une nouvelle orientation en émergence et qui bâtit à partir de ses acquis. Par cette contribution, nous souhaitons partager des modalités susceptibles d'aiguiller la structuration de la recherche en éducation au plan régional, selon une perspective multidisciplinaire, voire interdisciplinaire. En outre, nous désirons soumettre une articulation préliminaire d'orientations qui, à terme, permettront d'étudier des processus qui ont cours dans le cadre des activités d'un partenariat recherche-pratique.

Mots-clés : partenariat, recherche participative, collaboration, processus, pratique 


\section{REVUE HYBRIDE DE L'ÉDUCATION}

\section{Introduction}

Le Consortium régional de recherche en éducation du SaguenayLac-St-Jean (CRRE/02 ${ }^{1}$ ) est un partenariat recherche-pratique fondé en 1998. Par partenariat, nous référons à une structure associative permettant à des chercheurs et praticiens des établissements d'enseignement de s'engager à long terme. Les objets de travail y sont définis conjointement et les décisions sont partagées. Des rôles, des routines et des protocoles structurent les interactions entre les parties prenantes.

Le CRRE/02 est formalisé par un protocole d'entente signé par l'Université du Québec à Chicoutimi (UQAC), les quatre commissions scolaires $^{2}$ et les quatre cégeps ${ }^{3}$ de cette région.

Sa mission est de favoriser la compréhension, le développement, voire l'amélioration des pratiques professionnelles et ce qui en découle, aux divers ordres d'enseignement. Plus spécifiquement, le CRRE/02 poursuit les objectifs suivants :

- Accroître la présence d'une culture de recherche dans les milieux éducatifs

- Favoriser la concertation et l'harmonisation de tous les ordres d'enseignement

- Assurer une jonction entre les chercheurs et les intervenants du milieu scolaire

- Favoriser, par la recherche, la résolution de problèmes récurrents ou nouveaux, l'innovation et le développement de matériel pédagogique

- Prendre part au développement des collectivités régionales concernées

- Développer et diffuser des pratiques inspirantes pour alimenter le développement professionnel des communautés de pratique concernées

- Contribuer à la formation pratique des étudiants-chercheurs.

En premier lieu, ce texte rend compte de l'organisation d'un partenariat de recherche unique en éducation ainsi que de quelques-unes de ses réalisations. Ensuite, il positionne une orientation en émergence qui se construit à partir d'acquis historiques.

\footnotetext{
${ }^{1}$ Le nombre 02 fait référence au numéro de la région administrative du Saguenay-Lac-StJean.

${ }^{2}$ II s'agit des commissions scolaires des Rives-du-Saguenay, De La Jonquière, du Lac-StJean et du Pays-des-Bleuets.

${ }^{3}$ II s'agit des cégeps de Chicoutimi, de Jonquière et de St-Félicien ainsi que du Collège d'Alma.
} 


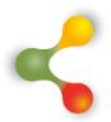

\section{REVUE HYBRIDE DE L'ÉDUCATION}

Par ce texte, nous souhaitons contribuer à l'identification de modalités susceptibles d'aiguiller la structuration de la recherche en éducation au plan régional, selon une perspective multidisciplinaire, voire interdisciplinaire. En outre, nous désirons soumettre une articulation préliminaire d'orientations qui, à terme, permettront d'étudier des processus qui ont cours dans le cadre des activités d'un partenariat recherche-pratique.

\section{La recherche participative en tant qu'orientation spécifique}

En comparaison avec d'autres entités de recherche dont la spécificité est fondée sur un thème de recherche, le dénominateur commun des activités du CRRE/02 réside dans l'approche de recherche participative adoptée. Un pan d'activités important consiste en la réalisation de projets de recherche qui émanent des priorités et défis définis avec les milieux scolaires. Le Consortium rassemble l'expertise requise pour traiter une variété de besoins et d'objets de recherche. Des projets visent à mettre en lumière des pratiques professionnelles inspirantes alors que d'autres ont une visée plus ou moins explicite d'amélioration des pratiques, en préconisant à la fois l'avancement des connaissances scientifiques ainsi que le développement professionnel des parties prenantes.

La perspective adoptée par rapport au concept d'amélioration des pratiques est de type non déficitaire. Nous entendons par là que les projets menés n'ont pas tant pour objectif de combler un écart par rapport à une norme dite probante ou un standard donné. Ils visent plutôt à mettre en place un dialogue entre recherche et pratique. Tout dépendant des contextes, des groupes et des individus, un tel dialogue peut mener à l'explicitation, à la confortation, au partage, à l'autorégulation, à l'abandon ou à la transformation de pratiques.

\section{La structure du partenariat}

Outre sa pérennité, une caractéristique importante d'un partenariat réside dans la formalisation de son fonctionnement. Au fil des années, différentes instances ont été mises en place à cet effet au sein du CRRE/02.

\section{Comité d'orientation}

Depuis sa création, le CRRE/02 dispose d'un comité d'orientation qui regroupe un représentant par organisation partenaire. Ce comité se réunit à quatre ou cinq reprises par année pour discuter des orientations générales du Consortium et en faire la gestion financière. La présidence est assumée en alternance par un représentant des commissions scolaires et des cégeps. La direction est quant à elle sous la responsabilité d'un professeur de I'UQAC. Le CRRE/02 dispose d'un 


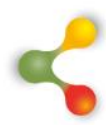

\section{REVUE HYBRIDE DE L'ÉDUCATION}

autofinancement annuel reposant sur une contribution provenant de chaque organisation partenaire.

Parmi les responsabilités spécifiques du comité d'orientation, on retrouve l'approbation des projets de recherche participative soumis par des équipes de chercheurs et de praticiens. Ces projets, qui sont soutenus financièrement à partir du fonds précédemment expliqué, sont préalablement évalués par un comité scientifique. Ce dernier est composé de chercheurs, de praticiens et d'étudiants-chercheurs. En plus de respecter les règles contemporaines de la recherche, les cinq à sept projets retenus annuellement répondent à un besoin des milieux de pratique et contribuent à l'avancement des connaissances et au développement professionnel. Le comité d'orientation approuve également des projets de recension des écrits, qui eux peuvent être soumis en tout temps.

Le comité d'orientation confie aussi le mandat de la journée annuelle scientifique du Consortium à un comité organisateur. Réunissant entre 70 et 100 participants - chercheurs, praticiens, étudiantschercheurs, membres de la communauté universitaire et régionale - cette journée est un lieu d'échange, de coordination et de mobilisation des connaissances issues des travaux du partenariat.

\section{Assemblée des chercheurs}

L'assemblée regroupe les chercheurs associés au Consortium. Elle permet à ses membres de discuter d'enjeux qui leur sont propres, eu égard au contexte du CRRE/02. Les réflexions de l'assemblée peuvent être acheminées au comité d'orientation par l'entreprise de son représentant qui y siège.

\section{Comité scientifique}

En plus de s'acquitter de l'évaluation des projets qui lui sont soumis, le comité scientifique formule occasionnellement des avis au comité d'orientation et à l'assemblée des chercheurs à partir de questions qui lui sont soumises. Il est aussi responsable de recommander au comité d'orientation l'octroi du statut de chercheur associé. Ce statut permet le dépôt d'une demande de financement. Un chercheur et son équipe l'obtiennent après avoir fait la démonstration au comité scientifique de sa compétence à mener un projet portant sur des questions d'éducation.

\section{Table des conseillers pédagogiques et des chercheurs}

Cette table est un lieu de concertation qui permet d'identifier des modalités pour favoriser une mobilisation élargie des connaissances issues des recherches du CRRE/02 ou d'autres provenances. Elle permet aussi une mise en commun des expertises régionales en matière de 


\section{8}

\section{REVUE HYBRIDE DE L'ÉDUCATION}

formation et de développement professionnel. La table est formée d'au moins un conseiller pédagogique par commission scolaire ou cégep et des membres de l'assemblée des chercheurs. Elle se réunit environ deux fois par année.

\section{Revue hybride de l'éducation}

La $\mathrm{RHE}^{4}$ est un moyen de diffusion en libre accès de travaux de recherche participative menés en sciences de l'éducation. Elle a comme particularité de combiner un volet scientifique et professionnel. Elle s'adresse donc autant aux chercheurs qu'aux praticiens de tous les ordres d'enseignement. Composée d'un comité éditorial international, la RHÉ respecte les principes d'évaluation par les pairs reconnus. Elle a aussi pour vocation de contribuer à la préparation de la relève scientifique en offrant un espace dédié aux étudiants-chercheurs et en leur permettant de s'approprier les rudiments de l'évaluation par les pairs et de la coordination d'une revue.

\section{Des retombées pour la pratique}

À ce jour, quelque 140 projets de recherche participative couvrant une diversité de champs disciplinaires ont été menés dans le cadre du partenariat CRRE/02.

Une analyse ${ }^{5}$ de 127 projets menés entre 1998 et 2018 a mis en lumière que l'orientation des projets vise majoritairement des retombées pour les praticiens des institutions d'enseignement. Plus précisément, et considérant qu'un projet peut poursuivre plus d'une visée, $61 \%$ des projets ciblent la transformation des pratiques, $22 \%$ permettent un développement d'instrument et $16 \%$ résolvent un problème de la pratique. En contrepartie, $36 \%$ des projets abordent la compréhension d'un phénomène, sans visée explicite ou implicite de changement.

\section{Vers une compréhension accrue de la dynamique collaborative et} partenariale

Un des atouts du CRRE/02 réside dans son approche de recherche participative. Elle combine à la fois l'avancement des connaissances scientifiques et le développement professionnel, ce qui rend la recherche utile aux yeux des praticiens et, bien évidemment, des chercheurs. En ce sens, il est peu surprenant de constater que les retombées visées ciblent le contexte de pratique.

\footnotetext{
${ }^{4}$ http://revues.uqac.ca/index.php/rhe/index

5 http://revues.uqac.ca/index.php/rhe/article/view/7/5
} 


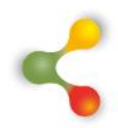

\section{REVUE HYBRIDE DE L'ÉDUCATION}

Même si le processus de travail, voire la dynamique des interactions entre participants, est sommairement documenté dans quelques recherches participatives - en particulier dans des recherches collaboratives - il convient de se pencher davantage sur la collaboration qui se met en place et se déploie dans le temps puisque nombreux sont les travaux qui se concentrent plutôt sur ce qui en découle.

\section{Des conditions favorables}

On identifie cinq conditions favorables au partenariat recherchepratique. Premièrement, pour définir l'objet de travail, il importe de considérer les caractéristiques du contexte local dans lequel le partenariat s'ancre, en particulier les besoins et problèmes de la pratique. Deuxièmement, chacune des parties prenantes doit percevoir un potentiel de retombées. La disponibilité de ressources, en particulier du temps, est une troisième condition cruciale. Une quatrième a trait à la qualité de la dynamique interactionnelle qui prend forme. Celle-ci se décline en cinq aspects : l'influence de l'attitude adoptée par les acteurs (particulièrement celles des chercheurs); l'identification et le partage de rôles; la mise à contribution active de l'ensemble des acteurs ; la nature du déroulement ou de la procédure d'animation retenue; la composition de l'équipe. La cinquième condition de succès concerne la présence d'un mécanisme de suivi du partenariat, notamment la pertinence d'un dispositif formel alimenté par des données.

\section{Définir une programmation autour de trois axes}

C'est dans l'intention d'approfondir la compréhension de la dynamique de travail entre chercheurs et praticiens que nous élaborons une programmation qui, en plus d'organiser le développement des connaissances, procurera des instruments et des méthodes de collecte et d'analyse de données pour porter un regard analytique sur toute forme de partenariat en éducation. C'est en quelque sorte un projet d'observatoire que nous ambitionnons fonder.

L'élaboration de la programmation, qui est soutenue par une subvention de soutien aux équipes du Fonds de recherche du Québec Société et culture (FRQSC), s'articule à partir d'une matrice combinant trois axes et trois niveaux d'action.

\section{Axes de recherche}

- Étude de la conception des activités du partenariat

- Étude de l'implantation, de l'évolution de ce qui se déroule lors des activités du partenariat

- Étude des retombées des activités du partenariat. 


\section{$\&$}

\section{REVUE HYBRIDE DE L'ÉDUCATION}

Niveaux d'action

- Individuel

- Collectif de travail

- Administration, gouvernance et communauté externe.

À terme, des objets de recherche ainsi que des instruments de collecte et d'analyse seront associés à chaque axe et niveau d'action pour permettre à des équipes de recherche de documenter des aspects relatifs aux processus de création et d'implantation/évolution des activités du partenariat, sans pour autant en négliger les retombées. II en découlera des connaissances sur divers processus, productions et savoirs qui prennent forme au cœur de la collaboration entre chercheurs et praticiens.

\section{Conclusion}

À l'heure où les enjeux de réussite éducative sont caractérisés par la complexité, le modèle du CRRE/02 offre une structure partenariale unique et durable des plus actuelles. Elle permet d'articuler de façon cohérente une concertation au niveau régional. La relation symétrique promue entre les parties prenantes s'appuie sur un dialogue qui combine une gamme de connaissances et de savoirs, tout en prenant en considération les spécificités des contextes. 